\title{
User Experience with a New Smartphone Application for Blood Glucose Monitoring (BGM) in an Information-Motivation -Behavioral Skills (IMB) Model Study
}

W. Fisher, PhD, FCAHS'; A. Stuhr, MD, MBA ${ }^{2 *}$; J. Wallace, BA, CCRA ${ }^{3}$;

S. Zhuplatov, MD, PhD³; T. S. Bailey, MD, FACE, CPI ; S. Pardo, PhD, PStat ${ }^{3}$

University of Western Ontario, Department of Psychology - Department of Obstetrics and Gynecology, London, Ontario, Canada; ${ }^{2}$ Ascensia Diabetes Care, Global Medical Affairs, Parsippany, NJ, USA; ${ }^{3}$ sscensia Diabetes Care, Global Clinical Affairs, Parsippany, NJ, USA; ${ }^{4}$ AMCR Institute, Office of the CEO, Escondido, CA, USA

*Presenting author

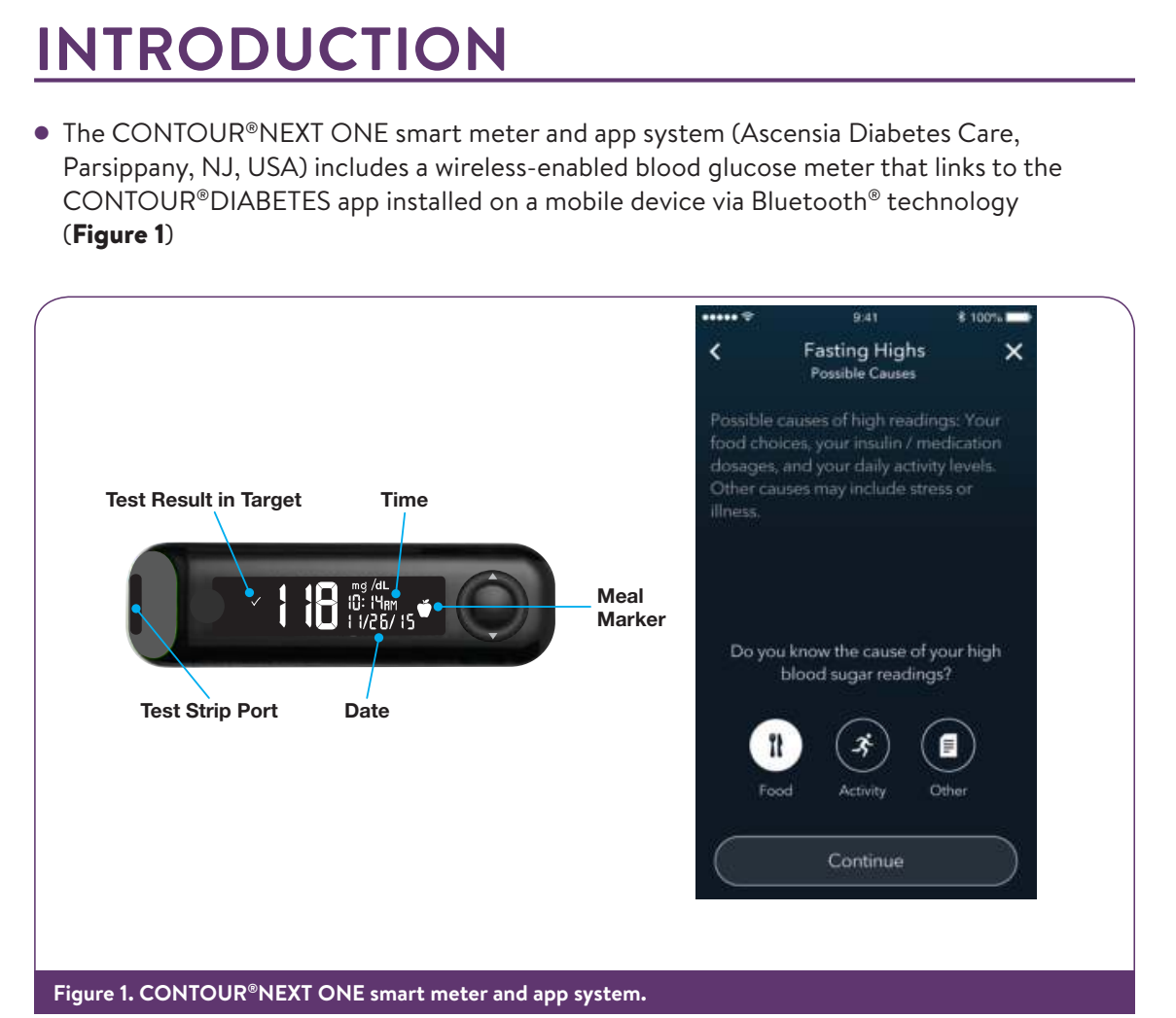

The CONTOURøDIABETES app (Version 2 Prototype) detects and reports patterns of blood

The app employs self-management feedback based on the
Behavioral Skills (IMB) model of health behavior change,

The IMB model is a well-researched and well-validated approach to health behavior change, which emphasizes the need for actionable information, motivation to act, and
behavioral skills for acting effectively to strengthen self-management of diabetes ${ }^{3.4}$

- The CONTOUR'NEXT ONE smart meter and app system is engineered to provide the user with actionable information, motivate the user
in applying skills in self-management action

- Features of the CONTOURøNEXT ONE smart meter and app system include My Readings notifications, and Smart Reming), blood glucose pattern detection with user-controlled blood glucose in different situations [referred to as Test Reminder Plans in the current version of the app]; Figure 2)

The blood glucose pattern notifications (e.g., Critical High, Running High, Critical Low,
Day of Week High) are user controlled; they provide messages that are informational (not prescriptive) and help the user consider the possible causes for blood glucose
patterns of concern and potential means for improving these outcomes

The CONTOUR NEXT ONE smart meter and app system also provides a user-controlled
opportunity for automatically checking back to see whether implemented changes have improved blood glucose outcom

a series of educational screens to identify the readings that triggered the pattern and

By decision of the user, recommen
related to the diagnosed pattern

- The CONTOUR'NEXT ONE smart meter and app system

- The current 6-week study with 46 insulin-using individuals with diabetes assessed the the functionality and self-management strength of the systere
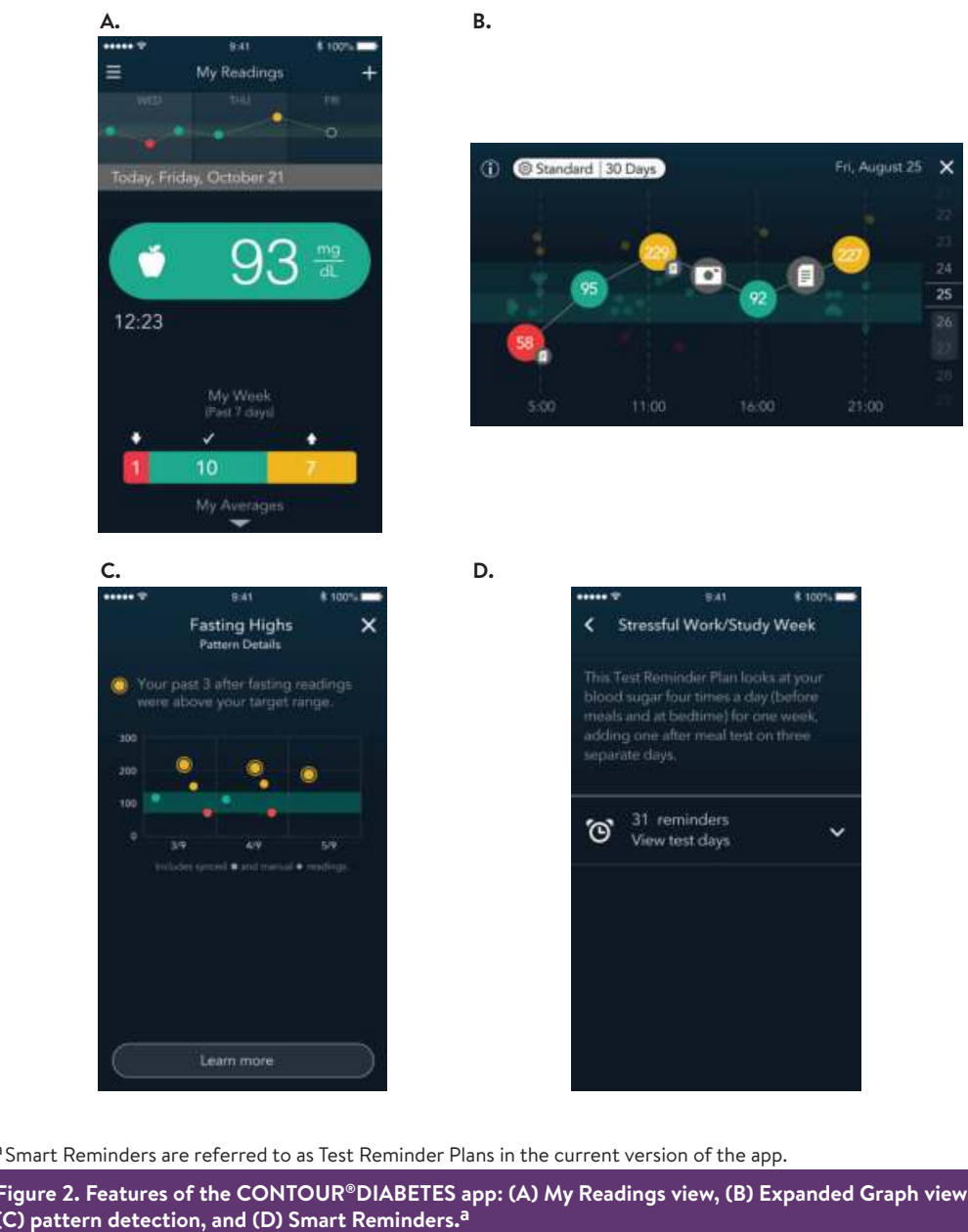

\section{OBJECTIVE}

To collect user experience observations concerning the CONTOUR ${ }^{\circledR}$ NEXT ONE smart

\section{METHODS}

\section{Study Design}

- This study was designed to explore participants' user experience with the

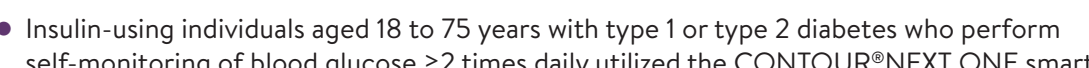

self-monitoring of blood glucose 22 times daily utilized the CON XUR'
meter and app system over a 6 -week period and reported their experiences on closed-

Each subject made two visits to the study site

- At Visit 1, subjects were given a brief overview of the CONTOUR ${ }^{\mathbb{N} E X X T}$ ONE smart

- Subjects downloaded the CONTOUR'DIABETES app (Version 2 Prototype) onto their nobile devices and were instructed
routine diabetes self-management

At Visit 2 , after 6 weeks of testing at home, subjects returned to the site, provided feedback via an interview with study staff, and completed questionnaires about their

Descriptive statistics were employed to characterize user experience with the

Primary Endpoint: Closed-ended Questionnaire Responses

- Closed-ended questionnaire items included 59 statements with responses based on

- Responses ranged from either $1=$ Strongly Agree to $5=$ Strongly Disagree,
or $1=$ Very Simple to $5=$ Very Difficult

A 'No Opinion' option was also offered

- The primary objective required that $285 \%$ of potential users should be able to perform the task (utilization of the app feature) suç
following four key statements trasks:

Obtain a synced blood glucose reading from meter to app

- Successfully access blood glucose displays, such as Expanded Graph and My Readings

views in the app

- Successfully access and use Smart Reminders in the app

- Additional questions assessed IMB model-specified information, motivation, and skill of
self-imanangementima app system

$$
\begin{aligned}
& \text { These questions are of special interesst and are featured in the following subsections by } \\
& \text { Cotegory: empowerment to self-manage, engagement and adherence, and the provision }
\end{aligned}
$$

category: empowerment ciss

\section{RESULTS}

Subjects

- 47 subjects with diabetes on insulin therapy enrolled in the study; 45 subjects completed
the study

\section{- One subject was w}

- Data from 46 subjects were andyzed including data from the subject who withdrew early Of these 46 subjects, $57 \%$ were female, $43 \%$ were male, $76 \%$ had type 1 diabetes, $24 \%$ had
type 2 diabetes, $39 \%$ used an insulin pump, and $61 \%$ used multiple daily injections Primary Objective

\begin{tabular}{|c|c|}
\hline \multicolumn{2}{|c|}{$\begin{array}{l}\text { The primary objective was met. Nearly all subjects reported successful syncing of the blood } \\
\text { glucose value on the meter with the appp, accessing and successfully interpreting blood } \\
\text { glucose displays, and accessing and using the Smart Reminders feature in the app (Table 1) }\end{array}$} \\
\hline Table 1. Questionnaire Results for the Primary Endpoint $(N=46)$ & \\
\hline Statement & $\begin{array}{l}\text { Subjects responding Agree, } \\
\text { Strongly Agree, or Neutral }\end{array}$ \\
\hline $\begin{array}{l}\text { Overall, I was able to successfully sync my } \\
\text { blood glucose value on the meter with the app }\end{array}$ & $89.1 \%$ \\
\hline $\begin{array}{l}\text { Overall, I was able to successfully access blood glucose } \\
\text { displays, such as Expanded Graph view and My Readings } \\
\text { view in the app }\end{array}$ & $100 \%$ \\
\hline $\begin{array}{l}\text { Overall, I was able to successfully interpret blood glucose } \\
\text { displays, such as Expanded Graph view and My Readings } \\
\text { view in the app }\end{array}$ & $97.8 \%$ \\
\hline $\begin{array}{l}\text { I was able to successfully access and use the Smart } \\
\text { Reminders feature in the app }\end{array}$ & $100 \%$ \\
\hline
\end{tabular}

IMB Foundations-Related Results

- Participant appraisal of the CONTOUR'NEXT ONE smart meter and app system was positive on muliple limB model dmensions, hiclading empowerment to seffmange the

Empowerment to Self-manage

- $73 \%$ of subjects Strongly
their diabetes (Figure 3 )

-74\% of subjects Strongly Agreed/Agreed that information learned through pattern-related messaging (e.g.
their diabetes

- Most subjects Strongly Agreed/Agreed that the blood glucose dis
Expanded Graph) were helpful to them in managing their diabetes

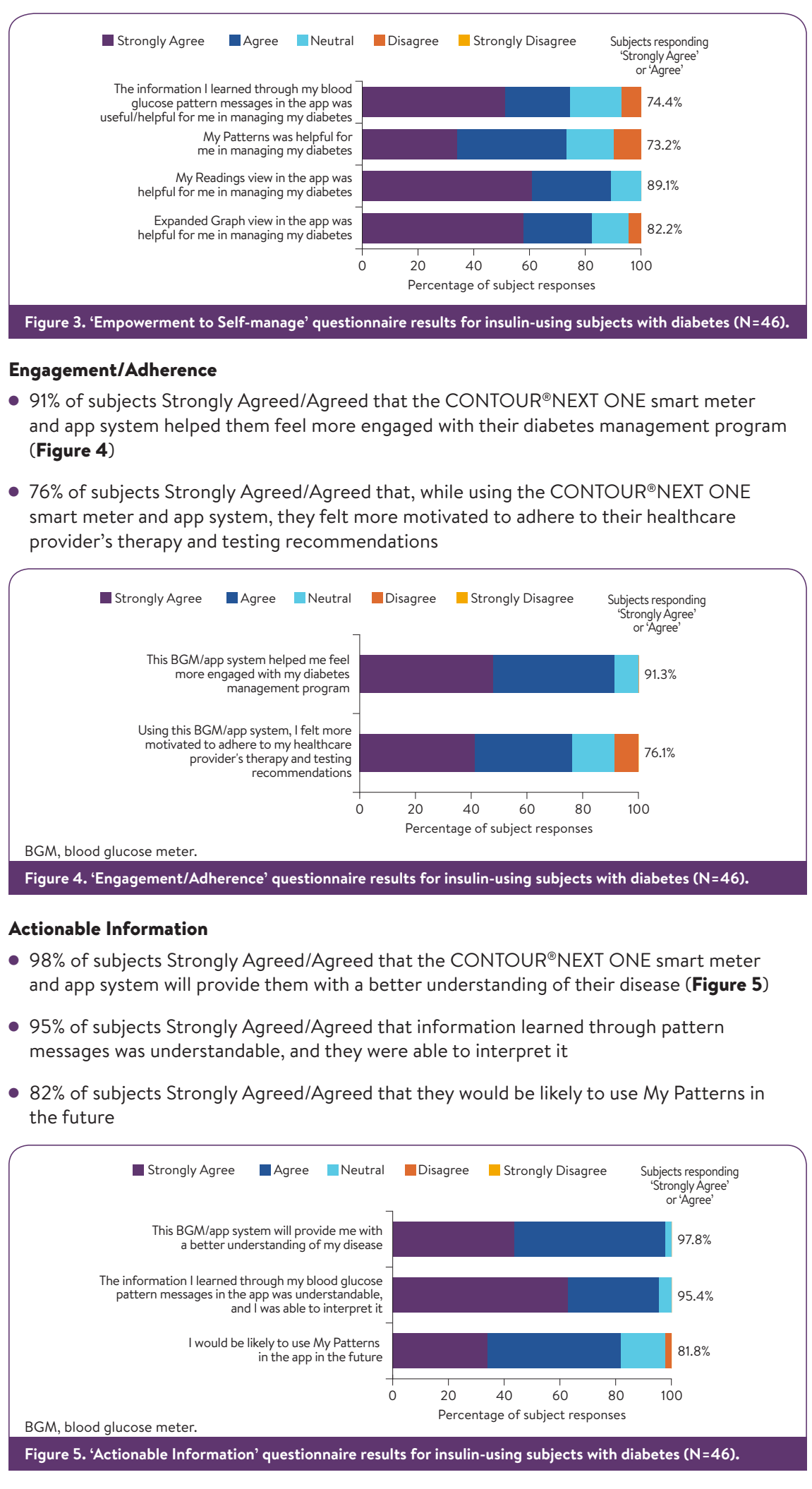

\section{DISCUSSION}

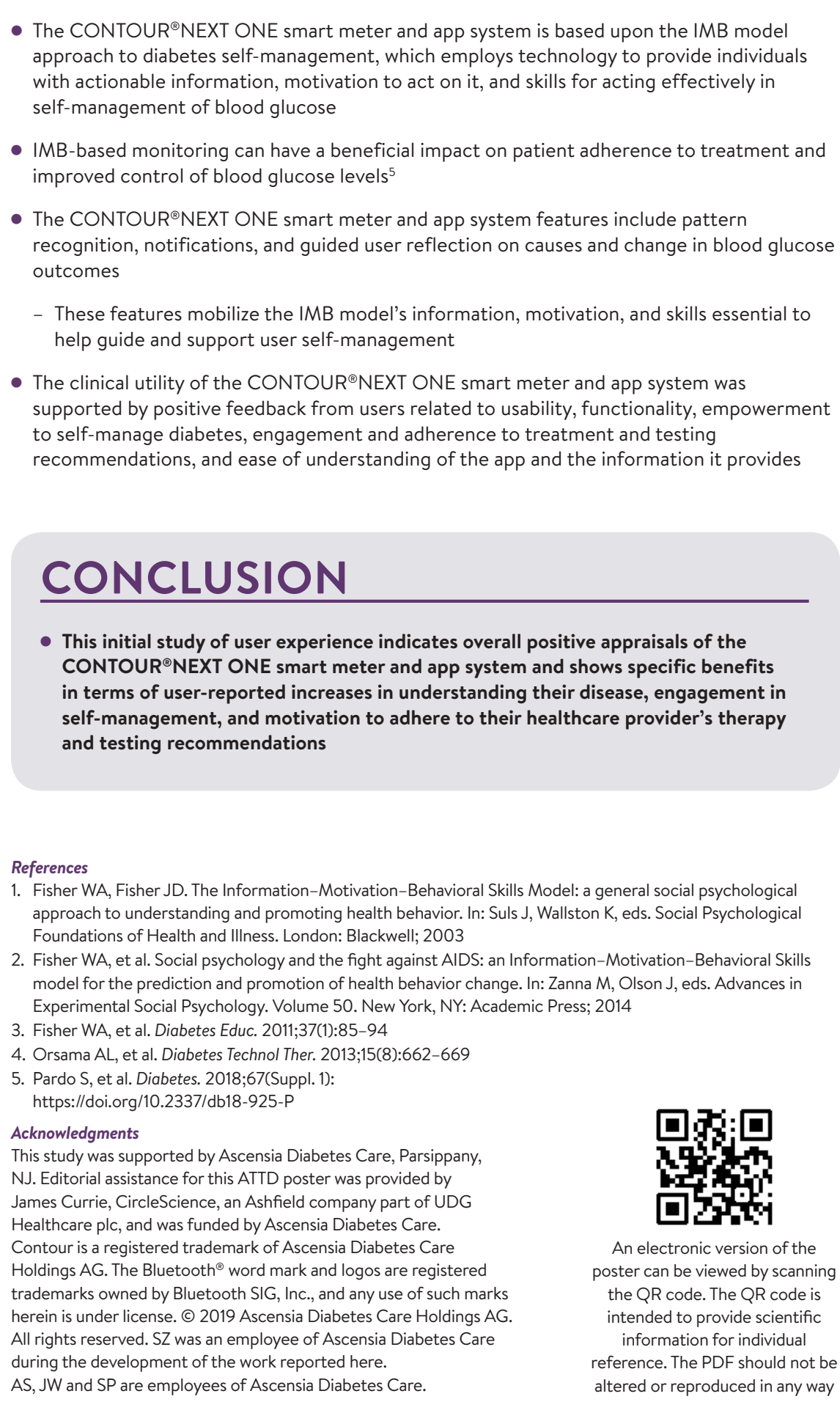

